\title{
Assessment of ciliary phenotype in primary ciliary dyskinesia by micro-optical coherence tomography
}

\author{
George M. Solomon, ${ }^{1}$ Richard Francis, ${ }^{2}$ Kengyeh K. Chu, ${ }^{3}$ Susan E. Birket, ${ }^{1}$ George Gabriel, ${ }^{2}$ \\ John E. Trombley, ${ }^{1}$ Kristi L. Lemke, ${ }^{2}$ Nikolai Klena, ${ }^{2}$ Brett Turner, ${ }^{1}$ Guillermo J. Tearney, ${ }^{3}$ \\ Cecilia W. Lo, ${ }^{2}$ and Steven M. Rowe ${ }^{1}$ \\ 'Department of Medicine, University of Alabama, Birmingham, Alabama, USA; Gregory Fleming James Cystic Fibrosis \\ Research Center, University of Alabama at Birmingham, Birmingham, Alabama, USA. ${ }^{2}$ University of Pittsburgh, \\ Pittsburgh, Pennsylvania, USA. ${ }^{3}$ Massachusetts General Hospital and Wellman Center for Photomedicine, Boston, \\ Massachusetts, USA.
}

Ciliary motion defects cause defective mucociliary transport (MCT) in primary ciliary dyskinesia (PCD). Current diagnostic tests do not assess how MCT is affected by perturbation of ciliary motion. In this study, we sought to use micro-optical coherence tomography ( $\mu \mathrm{OCT})$ to delineate the mechanistic basis of cilia motion defects of PCD genes by functional categorization of cilia motion. Tracheae from three PCD mouse models were analyzed using $\mu \mathrm{OCT}$ to characterize ciliary motion and measure MCT. We developed multiple measures of ciliary activity, integrated these measures, and quantified dyskinesia by the angular range of the cilia effective stroke (ARC). Ccdc39-/- mice, with a known severe PCD mutation of ciliary axonemal organization, had absent motile ciliary regions, resulting in abrogated MCT. In contrast, Dnah5 ${ }^{-/-}$mice, with a missense mutation of the outer dynein arms, had reduced ciliary beat frequency (CBF) but preserved motile area and ciliary stroke, maintaining some MCT. Wdr69-/- PCD mice exhibited normal motile area and CBF and partially delayed MCT due to abnormalities of ciliary ARC. Visualization of ciliary motion using $\mu O C T$ provides quantitative assessment of ciliary motion and MCT. Comprehensive ciliary motion investigation in situ classifies ciliary motion defects and quantifies their contribution to delayed mucociliary clearance.

Conflict of interest: The University of Alabama at Birmingham and Massachusetts Ceneral Hospital have filed for an unlicensed patent on the use of $\mu \mathrm{OCT}$ toward the functional imaging of respiratory mucosa, including for the use of high-throughput screening, estimation of rheology, and functional anatomy (e.g., cilia beating, airway surface liquid depth, and mucociliary transport) (US patent application 14/240,938). K.K. Chu, G.J. Tearney, and S.M. Rowe are named on the patent application.

Submitted: November 18, 2016 Accepted: January 19, 2017 Published: March 9, 2017

\section{Reference information:} JCI Insight. 2017;2(5):e91702. https:// doi.org/10.1172/jci.insight.91702.

\section{Introduction}

Primary ciliary dyskinesia (PCD) is a genetically heterogeneous autosomal recessive disease characterized by mutations that cause complete or partial ciliary immotility (1-5). Due to the prominent role of cilia in the airways, PCD-causing mutations result in abnormal mucociliary clearance, a primary defense of the respiratory tract, leading to sinus infection, progressive airway obstruction, and ultimately bronchiectasis and respiratory failure (5-7). Cilia dysfunction also adversely affects other organs that require motile cilia during organogenesis, including the brain $(8,9)$ and reproductive tracts $(10,11) .50 \%$ of PCD patients exhibit Kartagener's syndrome with situs inversus totalis (mirror symmetric visceral organ positioning), and $12 \%$ exhibit heterotaxy (randomized visceral organ situs) (12). Heterotaxy patients usually also exhibit complex congenital heart defects (12). Presently, over 30 PCD genes have been identified, but many more are likely to be discovered given the complexity of the ciliary machinery $(2,3,5,13)$.

The diagnosis of PCD is complex and currently involves multiple assessments that include genetic testing for mutations known to cause PCD, measurements of nasal nitric oxide (levels are very low in most forms of PCD for reasons that are not yet clear), electron microscopy to assess for ultrastructural defects of respiratory cilia, and high-speed video microscopy (HSVM) to detect abnormal respiratory ciliary motion (13). Patients are tested with a variety of these tests, making the diagnostic algorithm complex and challenging. Moreover, due to the lack of information regarding the functional consequences of many ciliary genes and multiple variants within any single gene that will vary in severity in an unpredictable fashion, it is useful to assess the functional consequences associated with suspected causes of PCD and begin to categorize the consequences of alleles in known PCD genes. While 
HSVM is clearly a useful tool, a means to evaluate ciliary motion in situ so that mucociliary transport (MCT) can also be measured would provide a major advance to improve functional characterization of the disease. The genetic heterogeneity, both in terms of the number of genes affected and the functional consequences of various mutations within any single gene, imposes important limitations on the utility of genetic testing. Cilia ultrastructural analysis has limited detection sensitivity, since not all PCD mutations cause cilia defects visualized by electron microscopy and the method is subject to artifacts. Functional analysis represents another important approach toward understanding ciliary motility (14-17), but this is technically challenging, and the relationship between ciliary motion and mucus transport has not been well characterized, since HSVM measurements are not conducted in situ $(13,18-21)$. For example, absence of motile cilia, reduced ciliary beat frequency (CBF), or abnormal ciliary stroke can each confer reduced mucus transport, but their relative importance to MCT is not fully understood and cannot be captured with HSVM alone (12). Recent work has demonstrated the capacity for HSVM to capture CBF and cilia waveform in submerged, excised nasal cells (21); however, these methods do not allow for in situ measures in the presence of native mucus and epithelial conditions. Hence, additional quantitative imaging tools are needed for the functional assessment of ciliary motion and the quantification of $\operatorname{MCT}(22,23)$ and could be a useful addition to the current tools available in the diagnostic armamentarium.

To begin to address these challenges, our laboratory has developed an innovative imaging technology, termed micro-optical coherence tomography ( $\mu \mathrm{OCT}$ ), a high-speed, high-resolution reflectance imaging modality, for obtaining cross-sectional images of the airway epithelium with a resolution of less than $1 \mu \mathrm{m}$ (24). This imaging modality can provide live real-time cross-sectional microscopy of the functional epithelial surface of living airways and epithelial monolayers. In the present study, we used three PCD mutant mouse models with differing levels of ciliary dysmotility, ranging from nearly immotile to active (dyskinetic), and then assessed CBF, ciliary stroke kinetics, and MCT rates. The findings suggest that $\mu \mathrm{OCT}$ imaging may be useful in the future for development as a diagnostic and phenotyping tool for PCD and other airway diseases likely to cause airway clearance defects.

\section{Results}

Metrics of ciliary motion. We previously showed that $\mu \mathrm{OCT}$, as opposed to conventional forms of OCT that have much lower resolution and imaging speed, can quantity a number of parameters relevant to the MCT apparatus, including periciliary layer depth, CBF, and MCT rate (25). To delineate ciliary defects more precisely, we optimized the $\mu \mathrm{OCT}$ technique to assess several additional parameters relevant to ciliary motion in general and PCD in particular (Figure 1). We measured the surface area of the epithelia that exhibits active ciliary motion (termed percentage motile cilia area), and using this measurement, calculated total ciliary workload, defined as the effective stroke rate $(\mathrm{ESR}=$ motile cilia area $\times \mathrm{CBF}$; Figure $1, \mathrm{~A}$ and B). As $\mu \mathrm{OCT}$ imaging allows visualization of cilia on individual cells and the ciliary stroke, which confers vectoral transport of the overlying mucus gel layers, we developed measures of individual ciliary patterns in 2 dimensions. This included quantifying the stroke distance (i.e., the ciliary length in the lateral direction) of an individual cilium in addition to the angular range of cilia (ARC) of the ciliary stroke, determined by tracing the coordinates of the arcs formed by the ciliary tip (Figure 1, A-C).

Analysis of the PCD mouse model harboring Ccdc39 mutation. To determine the effectiveness of $\mu \mathrm{OCT}$ imaging for quantitative assessment of ciliary motion characteristics and their effect on mucus transport, we conducted $\mu \mathrm{OCT}$ imaging of the respiratory epithelia from three different PCD mouse models previously recovered from a large-scale mouse mutagenesis screen (12). These PCD mutant mouse models exhibit a wide range of ciliary motion phenotypes, from largely immotile to relatively preserved ciliary motion, allowing us to discern how the $\mu \mathrm{OCT}$ signature relates to disease severity and included mice harboring pathogenic mutations in Ccdc39 (c.T2A; p.M1K), Dnah5 (c.5197C; p.S3350P), or Wdr69 (T10048C; p.L66P). Ccdc39 and Dnah5 are mutations known to cause PCD, while $W d r 69$ is a gene that has not been formally characterized but is predicted to cause PCD (12).

We first evaluated mice with a mutation in $C c d c 39$ (line $6261304 C l o$ ), a gene that causes severe PCD by disrupting microtubule assembly and axonemal organization of the cilium $(26,27)$. This mutant has a stop codon at the translation start site, causing a null phenotype. $C c d c 39^{-1-}$ mice can exhibit situs solitus (normal left-right patterning of visceral organs), situs inversus (mirror symmetric left-right patterning of visceral organs), or heterotaxy (randomized visceral organ patterning; Figure 2A). Those with heterotaxy invariably 

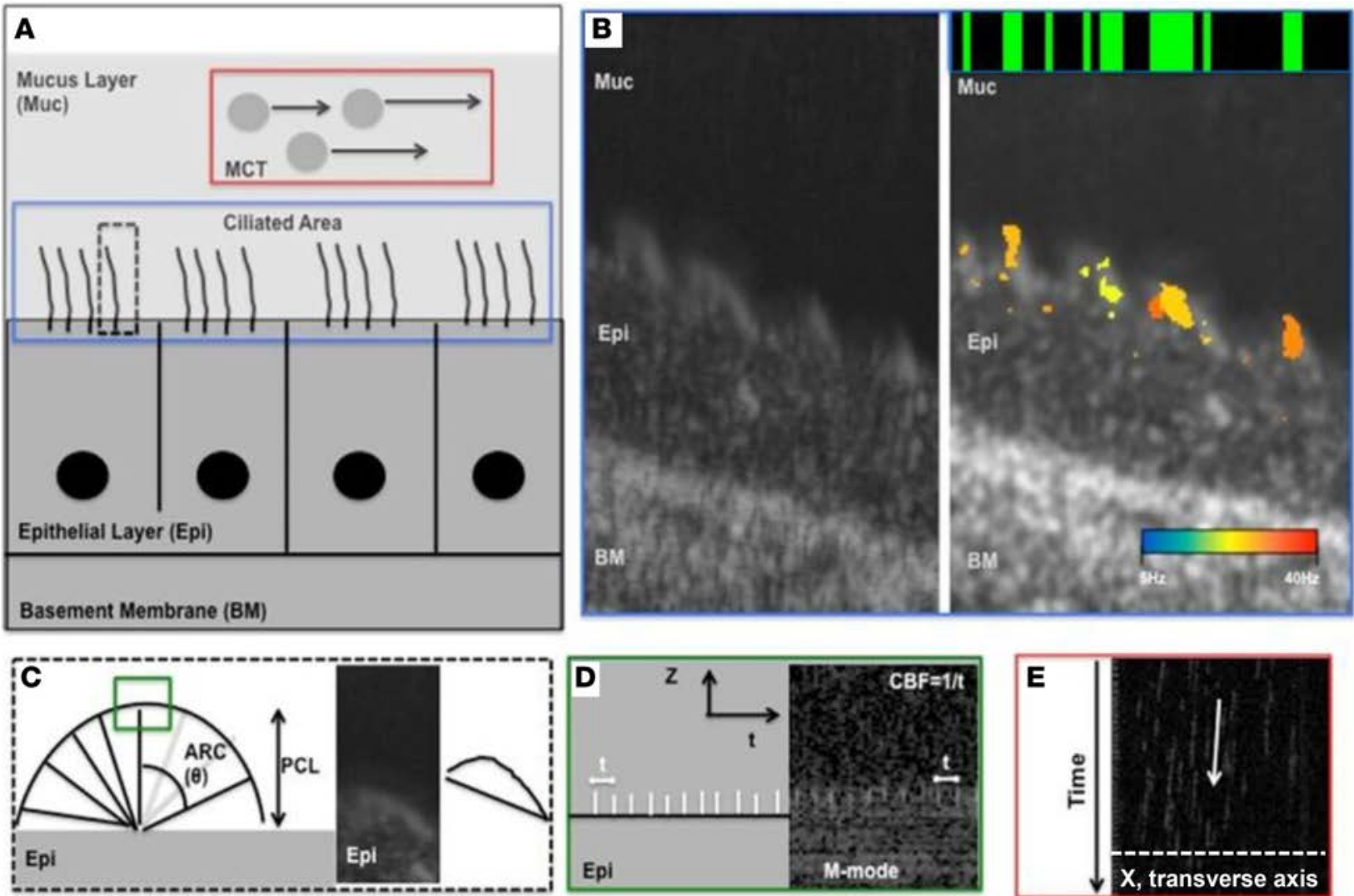

Figure 1. Representative schematic for methods of ciliary motion interrogation using $\mu \mathrm{OCT}$. (A) Schematic of epithelial cell surfaces, demarcating ciliated areas (blue box) and cilia arcs (black dashed box), and the mucus layer, with mucus transport (red box, Muc, mucus layer; BM, basement membrane; epi, epithelial layer; MCT, mucociliary transport rate). (B) Actual $\mu$ OCT of normal murine trachea (left). Coverage map of motile cilia areas in various CBFs, with the inset demonstrating that the percentage motile ciliated area is calculated by the ratio of active to inactive pixelated areas (right). (C) Representative cilia enlarged at full extent of cilia motion demonstrating ARC $(\theta)$. Periciliary layer (PCL) represents ciliary height. $\mu \mathrm{OCT}$ image (middle) of a cilia arc at full extension in the effective stroke and a tracing of the ciliary arc coordinates for calculation of ARC. (D) A schematic of the ciliary tip motion in the z-plane over time compared to an actual $\mu \mathrm{OCT}$ image of M-mode to determine CBF. Distance between z-plane spikes represents frequency of ciliary beat (CBF). (E) MCT is represented by the transport of particles over time across the transverse axis of the OCT beam (x).

die perinatally because of the presence of complex congenital heart defects. Homozygous $C c d c 39^{-1-}$ mice with situs solitus or situs inversus that do not exhibit congenital heart defects are initially viable. However, soon after birth they develop severe hydrocephalus and die, usually 5-7 days after birth. This is due to ependymal ciliary motion defects resulting in failure to maintain cerebral spinal fluid flow, causing closure of the cerebral aqueduct. Consistent with this, video microscopy of the trachea respiratory cilia showed mostly immotile cilia, with few cilia beating with slow, restricted, and dyskinetic motion (Supplemental Video 1; supplemental material available online with this article; https://doi.org/10.1172/jci.insight.91702DS1).

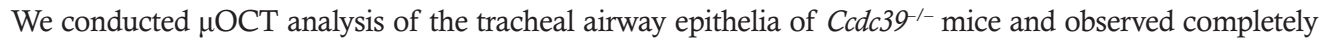
immotile cilia, except for a few residual cilia that exhibited slow, dyskinetic motion (Supplemental Video 2, right). In contrast, the tracheal epithelia of littermate controls showed rapid well-coordinated ciliary motion that swept across the respiratory epithelia (Supplemental Video 2, left). Quantitative $\mu \mathrm{OCT}$ analysis revealed the near absence of ciliated area, with active motion in $C c d c 39^{--}$mutant airway ( $<1 \%$ in mutant versus $17 \%-33 \%$ in control vertical a-lines with active ciliary motion, $P<0.005$; Figure 3 , A-C). Residual ciliary motion, which was scant, exhibited diminished CBF $\left(31.8 \pm 5.4 \mathrm{~Hz}\right.$ for $C c d c 39^{+/+}$versus $3.4 \pm 2.2 \mathrm{~Hz}$ for $C c d c 39^{--}$; Figure 3D) and was dyskinetic, with a severely narrowed $\mathrm{ARC}\left(61.4^{\circ} \pm 5.04^{\circ}\right.$ for $C c d c 39^{+/+}$versus $15.38^{\circ} \pm 2.58^{\circ}$ for $C c d c 39^{-/-}$, $P<0.0001$; Figure $3 \mathrm{~F}$ ). There was a severe reduction in ESR, an integrated measure of $\mathrm{CBF}$ and ciliated area (5.38 \pm 1.50 for $C c d c 39^{+/+}$versus $0.01 \pm 0.2$ for $C c d c 39^{-/}, P<0.05$; Figure $3 \mathrm{E}$ ). Together, these alterations would predict severely diminished transport capacity. This was confirmed with the observation of near-complete loss of MCT upon addition of a standardized airway liquid to control for any acquired abnormality of mucus $(0.43$ $\pm 0.06 \mathrm{~mm} / \mathrm{min}$ for $C c d c 39^{+/+}$versus $0.01 \pm 0.003 \mathrm{~mm} / \mathrm{min}$ for $C c d c 39^{--}, P<0.005$; Figure 3 , G-I). These find- 

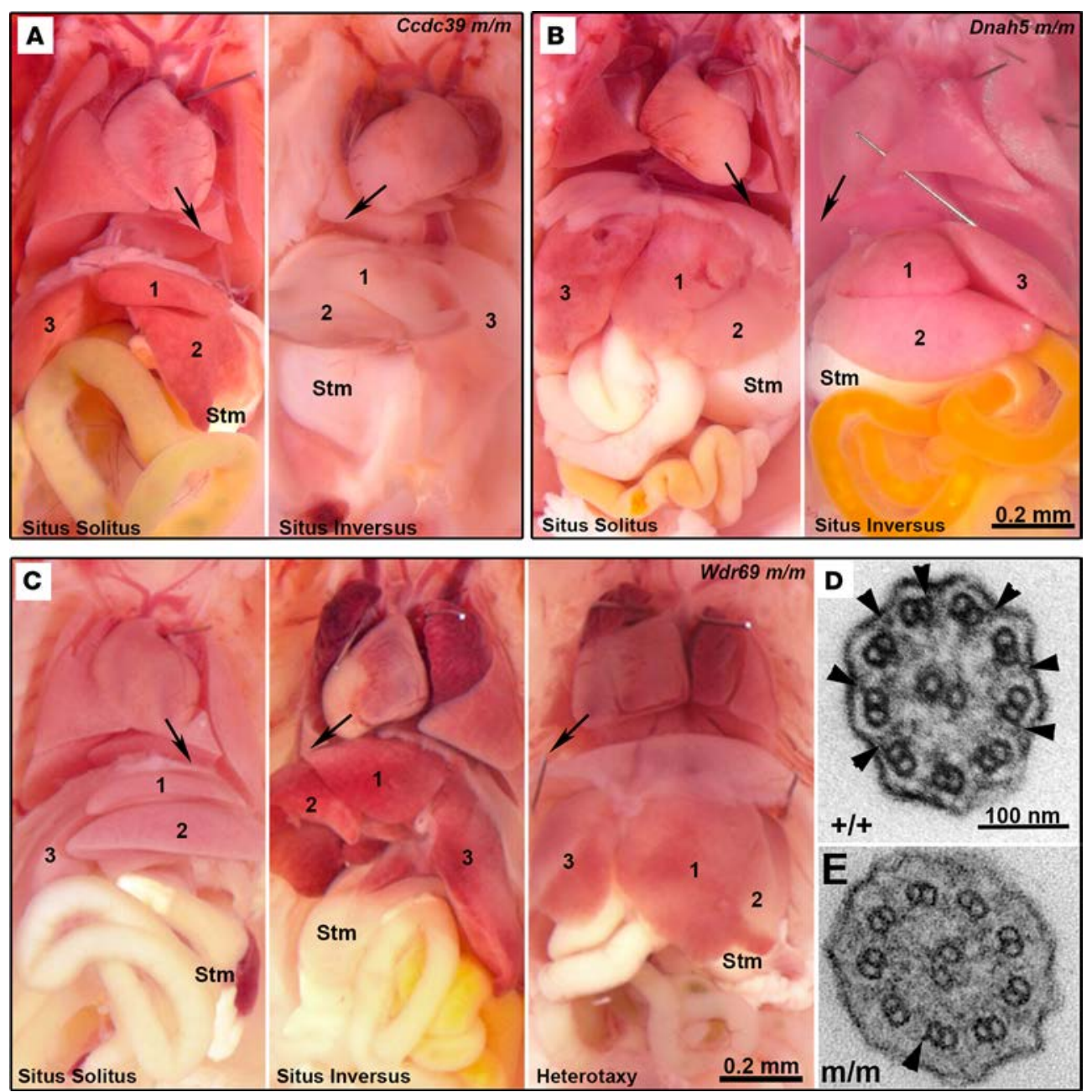

Figure 2. Pathological characterization of PCD mice. (A) Representative pathological sections from $\mathrm{Ccdc39} 9^{-/-}$ mice $(n=5)$. (B) Representative pathological sections from Dnah5 $5^{-/}$mice ( $n$ = 5). (C) Representative pathological sections from Wdr69-- mice. (A-C) Situs inversus is demonstrated when the cardiac axis is rightward and the spleen and liver are reversed in the abdomen. Number designations indicate organ laterality (1, lobes of the liver; 2 , the hepatic vein; 3 , the spleen). Black arrows indicate the axis of the heart. Stm, stomach. Scale bar: $0.2 \mathrm{~mm}$. (D) Representative electron micrograph of cilium indicating normal expression of outer dynein arms (black arrowheads, $n=10$ ) in Wdr69 $9^{+/+}$mice compared with (E) Wdr69 ${ }^{-/-}$mice, demonstrating reduced expression of outer dynein arms. Scale bar: $100 \mathrm{~mm}$.

ings indicate that reduced motile ciliary coverage, combined with other defects, such as residual dyskinetic ciliary motion and narrow ARC, underlie the near-complete loss of mucus transport in the $\mathrm{C} c d c 39^{-1}$ airway (shown as percentage of control in Table 1 in comparison to other mutations). This is consistent with the severe phenotypes observed in $C c d c 39^{-1-}$ mice (Table 1) and poor clinical outcomes associated with PCD patients harboring CCDC39 mutations in comparison to other PCD variants $(27,28)$.

Analysis of PCD mouse model harboring a mutation in Dnah5. We next evaluated Dakshi Dnah5 (b2b016Clo) mutant mice. Dnah 5 encodes a motor protein of the outer dynein arm (ODA) of motile cilia and is a gene commonly found to cause PCD (29). Dakshi mutants, like other Dnah5 and PCD mutants, exhibited situs inversus, situs solitus, or heterotaxy (see Figure 2B) but, as their mutation is caused by a missense mutation, Dakshi mutants represent a mild genetic variant. Consequently, some homozygous Dakshi mice can survive into adulthood without overt hydrocephalus. This is likely explained by preserved ciliary motion in Dakshi mutant airways (see HSVM image of Dakshi mutant, Supplemental Video 3). Using $\mu$ OCT imaging of excised trachea from postnatal day 20, homozygous Dakshi mice (see Supplemental Video 4, right) had preserved ciliary motion, a pattern not dissimilar to that seen in wild-type littermate controls (Supplemental Video 4, left; Figure 4, A-C). However, quantitative $\mu$ OCT analysis showed a $64 \%$ reduction in CBF in the

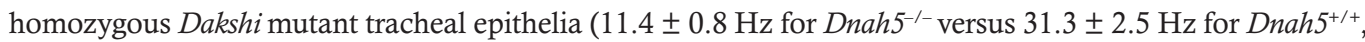
$P<0.0001$; Figure 4G). The cilia frequency spectrum also appeared altered (Figure 4, D and E), although

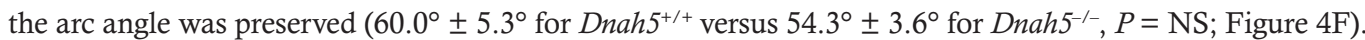
Reduced CBF combined with diminished expression resulted in a marked reduction of ESR (Figure $4 \mathrm{H}$ ). Importantly, mucus transport with standardized airway liquid showed a $65 \%$ reduction in MCT $(0.43 \pm 0.06$

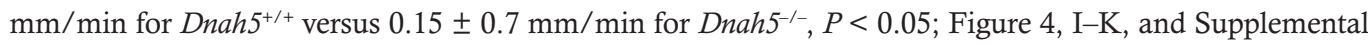
Video 5). Together these findings suggest that the reduction in mucus transport in the Dakshi mutants is principally due to reduced CBF rather than an altered motion pattern of individual cilia. $\mu O C T$ readily distinguished the functional basis of ciliary motion abnormalities in the $C c d c 39$ mutants versus the Dakshi mutants and also quantified their severity, demonstrating retention of $50 \%$ of the MCT function in the Dakshi mutant but less than $1 \%$ of MCT capacity in Ccdc39 (Table 1 ). 
Table 1. Quantitative assessment of respiratory cilia function and mucus transport

\begin{tabular}{|c|c|c|c|c|c|c|c|c|c|c|}
\hline \multirow{4}{*}{$\begin{array}{l}\text { Mutation } \\
\text { Ccdc39 }(n=5)\end{array}$} & \multirow{2}{*}{\begin{tabular}{|c|} 
Genotype \\
Mutant
\end{tabular}} & \multirow{2}{*}{$\begin{array}{c}\text { Motile cilia area } \\
\text { (\% of control) } \\
4.25 \%\end{array}$} & \multicolumn{2}{|c|}{$\begin{array}{c}\text { Ciliary beat } \\
\text { frequency }(\mathrm{Hz})\end{array}$} & \multicolumn{2}{|c|}{ Arc angle $\left({ }^{\circ}\right)$} & \multicolumn{2}{|c|}{ Effective stroke rate } & \multicolumn{2}{|c|}{$\begin{array}{l}\text { Mucus Transport } \\
\text { (mm/min) }\end{array}$} \\
\hline & & & 3.4 & $11.0 \%$ Ctrl & 15.4 & $25.1 \%$ Ctrl & 0.01 & $<1 \%$ Ctrl & 0.01 & N/A \\
\hline & Ctrl & & \multicolumn{2}{|c|}{30.8} & \multicolumn{2}{|l|}{61.4} & \multicolumn{2}{|c|}{5.4} & \multicolumn{2}{|l|}{0.43} \\
\hline & & $P<0.005$ & \multicolumn{2}{|c|}{$P<0.005$} & \multicolumn{2}{|c|}{$P<0.0001$} & \multicolumn{2}{|c|}{$P<0.005$} & \multicolumn{2}{|c|}{$P<0.005$} \\
\hline \multirow[b]{2}{*}{$\operatorname{Dnah5}(n=5)$} & Mutant & $43 \%$ & 11.4 & $36.3 \% \mathrm{Ctrl}$ & 54.3 & $90.5 \% \mathrm{Ctrl}$ & 1.10 & $21.8 \%$ Ctrl & 0.15 & $34 \%$ Ctrl \\
\hline & Ctrl & & \multicolumn{2}{|l|}{31.3} & \multicolumn{2}{|l|}{60.0} & \multicolumn{2}{|l|}{5.04} & \multicolumn{2}{|l|}{0.43} \\
\hline \multirow[t]{2}{*}{ Wdr69 $(n=8)$} & Ctrl & & 23.5 & & 60.9 & & 3.0 & & 0.45 & \\
\hline & & NS & \multicolumn{2}{|c|}{ NS } & \multicolumn{2}{|c|}{$P<0.0005$} & \multicolumn{2}{|c|}{ NS } & \multicolumn{2}{|c|}{$P<0.05$} \\
\hline
\end{tabular}

$\mathrm{Hz}$, beats/min; ND, none detected; Ctrl, control.

Analysis of PCD mouse model harboring mutation in Wdr69. We next examined mice harboring mutations in $W d r 69$ (b2b1304Clo), a novel gene predicted to be a protein required for ODA assembly based on sequence comparison with Chlamydomonas $(30,31)$. This was confirmed with cilia ultrastructural analysis by electron microscopy, which showed the absence of ODA in the respiratory cilia of the tracheal epithelia of $W d r 69^{-1}$ mice (Figure 2, D and E). Wdr69-/- mutants had the same spectrum of laterality phenotypes as observed in other PCD mice, including situs solitus, situs inversus, or heterotaxy with complex congenital heart defects

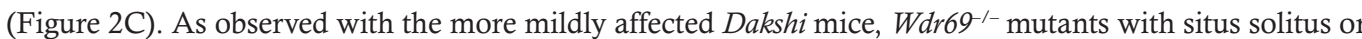
situs inversus can survive postnatally, but some mutants developed hydrocephalus and died at 2-3 weeks of

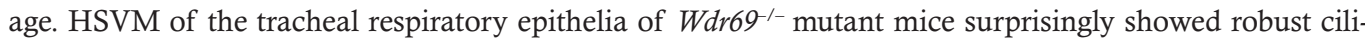
ary motion (see Supplemental Video 6). To further characterize ciliary motion, we conducted quantitative $\mu \mathrm{OCT}$ image analysis. No significant difference was observed in the degree of ciliated area $(12.5 \% \pm 3.0 \%$

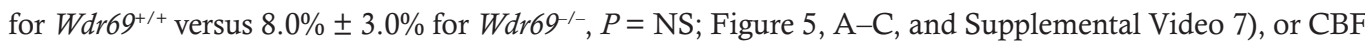
$\left(23.5 \pm 1.2 \mathrm{~Hz}\right.$ for $W d r 69^{+/+}$versus $17.9 \pm 3.4 \mathrm{~Hz}$ for $W d r 69^{--,} P=\mathrm{NS}$; Figure $\left.5 \mathrm{D}\right)$. As a result, there was no significant difference in the ESR $\left(3.0 \pm 0.5\right.$ for $W d r 69^{+/+}$versus $1.4 \pm 0.4$ for $W d r 69^{-/-}, P=$ NS; Figure $5 \mathrm{E})$. However, MCT was significantly depressed in $W d r 69^{-/-}$mice $\left(0.45 \pm 0.07 \mathrm{~mm} / \mathrm{min}\right.$ for $W d r 69^{+/+}$versus $0.24 \pm 0.08 \mathrm{~mm} / \mathrm{min}$ for $W d r 69^{-/-}, P<0.05$; Figure $5 \mathrm{~F}$ and Supplemental Video 8 ). The ciliary motion in the $W d r 69^{-1-}$ mutant appeared dyskinetic by $\mu \mathrm{OCT}$, suggesting it may be inadequate to transfer motile force to the overlying mucus (Figure 5, G-I). Examination of the ciliary frequency spectral pattern showed minimal changes (Figure 5, J and K), but an abnormal stroke pattern was evident, with a marked reduction in the ARC $\left(\theta=60.0^{\circ} \pm 5.3^{\circ}\right.$ for $W d r 69^{+/+}$versus $20.6^{\circ} \pm 4.2^{\circ}$ for $W d r 69^{--}, P<0.0005$; Figure $\left.5, \mathrm{~L}-\mathrm{N}\right)$. This was confirmed by HSVM (Figure $5 \mathrm{~N}$ ). These results indicate that $\mathrm{Wdr69^{-/- }}$ mutants exhibit mild PCD due to abnormal ciliary motion, despite normal ciliated area and CBF, which confers a slight reduction in MCT due to suboptimal translation of ciliary energy to the overlying mucus (Table 1). These results further demonstrated the power of an integrated analysis of ciliary function afforded by $\mu$ OCT for phenotyping ciliary dynamics.

\section{Discussion}

The diagnosis and risk stratification of PCD remains problematic as new genes and mutations that cause PCD continue to emerge. Here, we show the efficacy of $\mu \mathrm{OCT}$ imaging to provide an integrated quantitative assessment of the functional parameters of ciliary motion and mucus transport to characterize respiratory ciliary function in three PCD mutant mice. Using $\mu \mathrm{OCT}$ imaging, we readily obtained multiple integrated parameters of ciliary expression, beating, and motion pattern and linked abnormal ciliary motion to defective MCT to functionally categorize the nature of the abnormality. To support this, we examined integrative quantitative parameters for evaluating ciliary motion characteristics by $\mu \mathrm{OCT}$ : motile cilia area, ESR, and ARC. We further showed that $\mu \mathrm{OCT}$ analysis could characterize a mutation predicted to cause PCD, clearly identifying the manner by which it disrupts ciliary motility. To our knowledge, this is the first report of a technology capable of in situ imaging that can characterize ciliary motion and mucus transport simultaneously. 

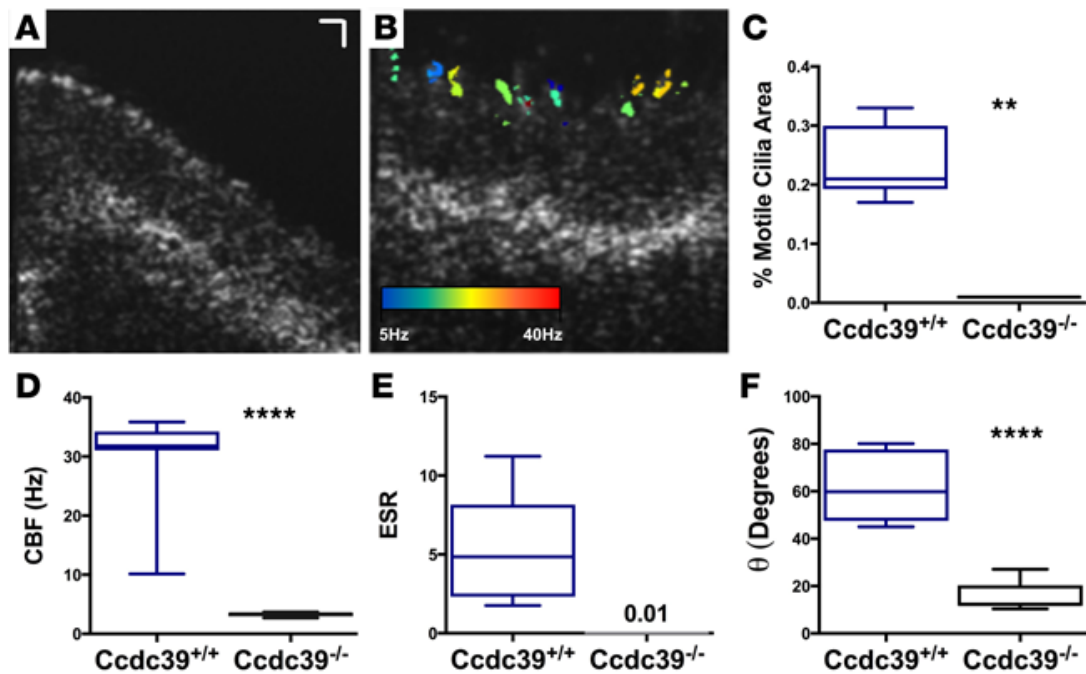

G

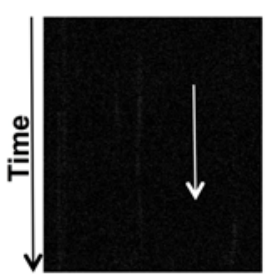

H

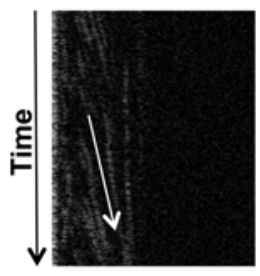

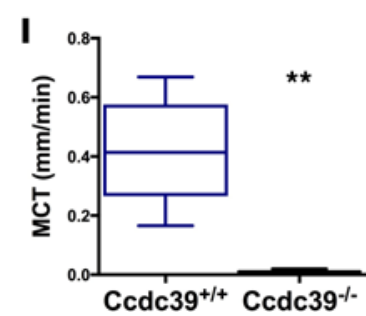

Figure 3. Characterization of $C c d c 39^{-/-}$mice. (A) Representative motile cilia area map from $\left[c d c 39^{-/-}\right.$tracheae at 5 days old indicates near absence of active ciliary movement in the mutant $(n=5)$. (B) Representative

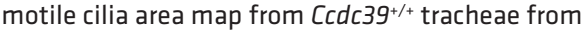
a littermate control. Colored pixels indicate areas of active cilia movement at varied CBFs $(n=5)$. (C) Quantification of reduced percentage of motile ciliated areas in $C c d c 39^{-1-}$ mice. ${ }^{* *} P<0.005$. (D) Ciliary activity maps indicates undetectable (ND) CBFs in $\mathrm{Ccdc} 39^{-/-}$mice. ${ }^{* * *} P<0.0001$. (E) ESR indicates that the reduced MCT in this mutant class is due to absent ciliary movement and reduced ciliary expression. (F) Residual motile cilia demonstrate dyskinesia with reduced arc angle in $C c d c 39^{-/-}$mice. ${ }^{* * *} P<0.0001$. (G and $\mathbf{H}$ ) Absent fluid transport is observed in $\mathrm{Ccdc}_{\mathrm{C}} \mathrm{9}^{-/-}$mice (G) compared with controls (H). (I) MCT is nearly absent in Ccdc39-/- mice due to effectively reduced ciliated area and movement. ${ }^{* *} P<0.005 . n=6\left[c d c 39^{+/+}\right.$animals and 5 Ccdc39 ${ }^{-/-}$animals; 4 ROIs per trachea. All data are represented as mean (line) with minimum and maximum boundaries displayed. Statistical significance determined by Mann-Whitney $t$ test. Scale bar: 10 microns (two dimensions, $\mathbf{A}$ and $\mathbf{B}$ ); 10 micron by 10-second images ( $\mathbf{G}$ and $\mathbf{H}$ ).

Our findings showed that reduced MCT can arise from different etiologies, such as the absence of motile cilia, cilia with reduced CBF, and/or cilia with abnormal stroke pattern. Changes in these parameters individually or in combination can affect MCT and, ultimately, affect mucociliary clearance of the lung. The MCT reduction in the $C c d c 39$ mutant was principally due to the effects of marked reduction in the motile ciliated area. In contrast, in the Dakshi Dnah5 mutant, reduced CBF was observed without detectable changes in either motile ciliated area or ARC, resulting in a more mild reduction in MCT (65\% reduction versus control, as compared with complete disruption in $\left.C c d c 39^{--}\right)$. Interestingly, in the Wdr69 mutant, no significant change was observed for the motile cilia area and only mild reductions were observed in $\mathrm{CBF}$, consistent with a less severely affected ciliary motion apparatus, as suggested by HSVM. Consequently, MCT was only somewhat reduced (69\% reduction compared with littermate controls) and was caused by reduced ARC. A functional approach enabled by $\mu \mathrm{OCT}$ allows an integrated measurement of ciliary function and mucus transport that will be highly useful to the field, since the severity of functional defects cannot always be predicted by the genetic analysis due to the large number of genes affected and the variable underlying severity of the gene defect (i.e., missense versus nonsense alleles).

Overall, the severity of the MCT defect measured by $\mu \mathrm{OCT}$ reflected the importance of the ciliary motion abnormality in regards to the transport of mucus. The severity of the MCT defect was also correlated with the severity of hydrocephalus in the mutant mice. $C c d c 39$ mutants exhibited the slowest MCT and the most severe hydrocephalus (mice did not survive beyond 7 days after birth), whereas Dnah5 and Wdr69 mice exhibited a less severe phenotype (with prolonged survival). These findings are relevant to the recent report of more severe lung disease in patients with nonsense mutations of $C C D C 39$, an important clinical finding whose explanation was not previously apparent $(27,28)$. The alterations in ciliary parameters detected by $\mu \mathrm{OCT}$ imaging may have relevance for driving therapeutic decisions. For example, those with normal ciliary stroke but reduced $\mathrm{CBF}$ may respond to $\mathrm{CBF}$ agonists, but this approach may not be efficacious in patients with a severe deficiency in motile cilia that will require other approaches to restore completely absent ciliary motility.

Here, we have also described a gene predicted to cause PCD - Wdr69, an ODA protein. We observed abnormal ARC in this missense mutation that reduced energy transfer from the cilia to the mucus layer, explaining the divergence in MCT and ESR measurements. Cilia ultrastructural electron microscopy analysis of the Wdr69 mutant airway revealed an ODA defect, consistent with the known role of $W d r 69$ based on the Chlamydomonas homolog (30). Based on this analysis, we expect WDR69 is a gene capable of causing 


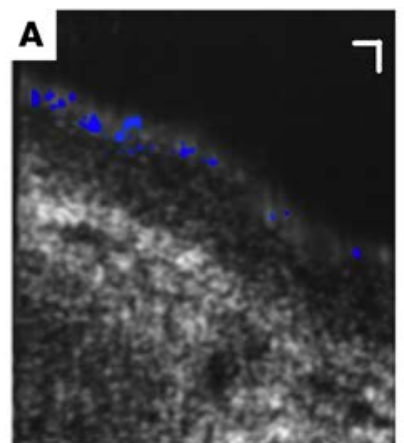

D

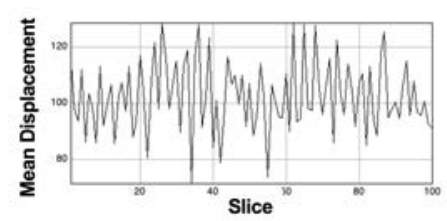

G

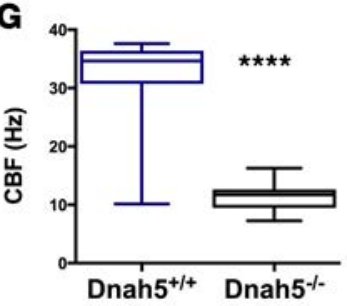

I

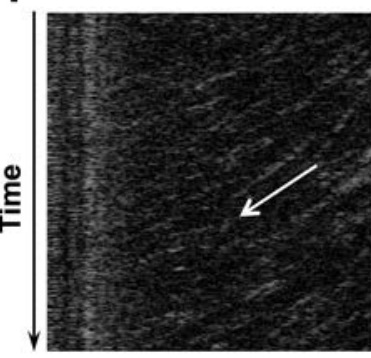

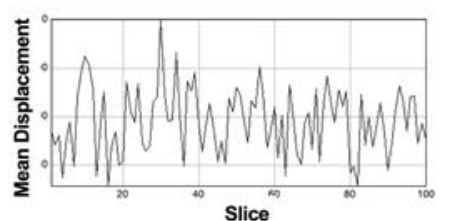

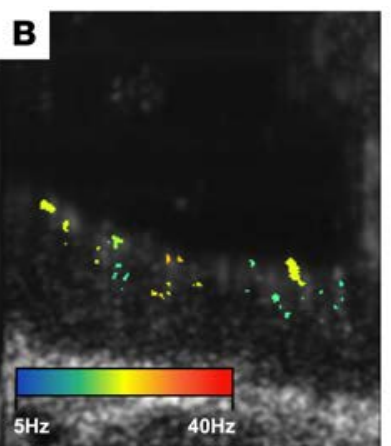

E

H
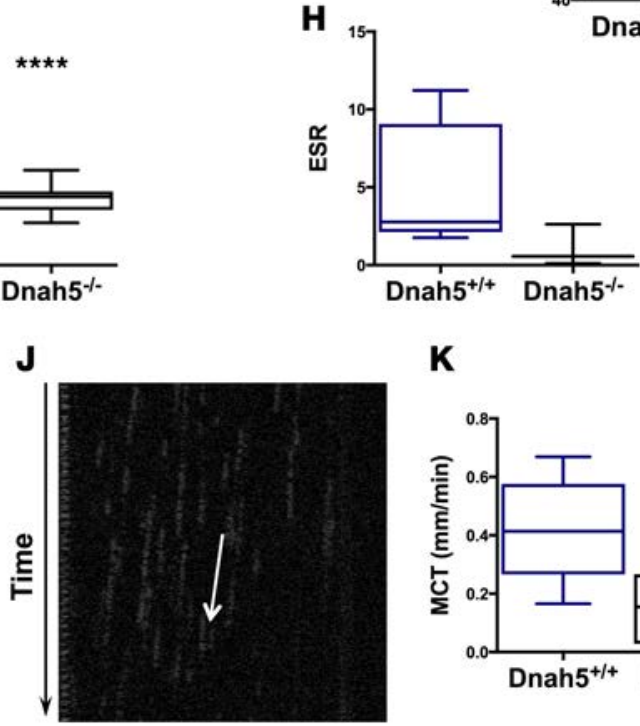

K

C

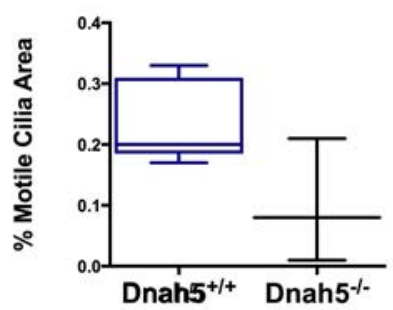

F
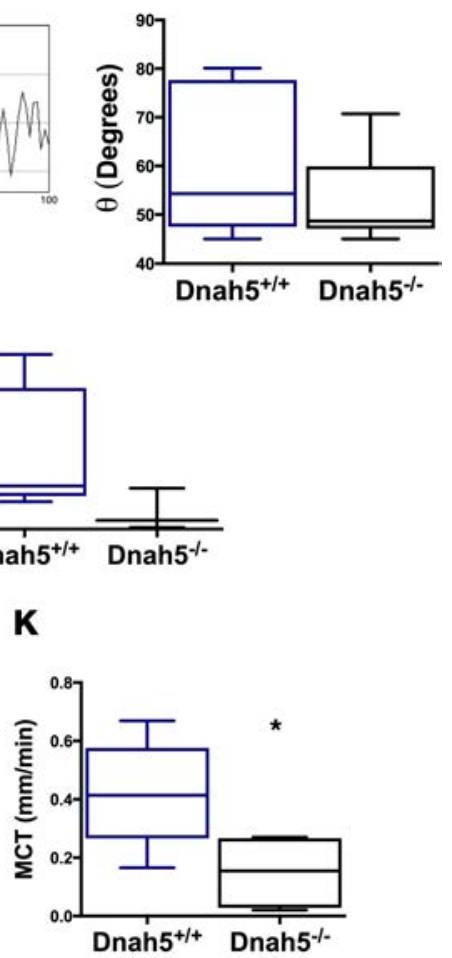

Figure 4. Characterization of Dnah5 ${ }^{-/-}$ mice. (A) Representative motile cilia area map from Dnah5 $5^{-/-}$mice indicates robust ciliary motion but at a lower CBF than demonstrated in B. (B) Representative ciliary motile area map from $\mathrm{Dnah5}^{+/+}$littermate control mice $(n=5)$. Colored pixels indicate areas of active cilia movement at varied CBFs. (C) Quantification of the percentage of motile ciliated areas in

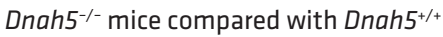
littermate control mice. $P=$ NS. (D and $\mathbf{E}$ ) Representative waveform plots from Dnah5 $5^{+/+}$and Dnah5 $5^{-/-}$mice, respectively, indicating diminished ciliary activity at the ROI examined ( $n$ = 5 mice). "Slice" refers to the ROI of ciliary displacement. (F) Cilia demonstrate normal arc angle. $P=$ NS. (G) CBF is reduced in Dnah5 $5^{-1-}$ mice compared with Dnah5 ${ }^{+/+}$littermate control mice. ${ }^{* * *} P<0.0001$. (H) Reduced CBF results in diminished ESR in Dnah5 ${ }^{-/-}$mice. (I and J) Reduced fluid transport is observed in Dnah5 $5^{-/-}$mice (I) compared with controls (J). (K) As a result of reduced CBF but not reduced ciliated area, Dnah5 ${ }^{-1-}$ mice demonstrate impaired mucociliary clearance. ${ }^{*} P<0.05 . n=6$ Dnah5 $^{+/+}$animals and 5 Dnah5 ${ }^{-/-}$animals; 4 ROls per trachea. All data are represented as mean (line) with minimum and maximum boundaries displayed. Statistical significance determined by Mann-Whitney $t$ test. Scale bar: 10 microns (two dimensions, $\mathbf{A}$ and $\mathbf{B}) ; 10$ micron by 10 -second images (I and J).

PCD; however, mutations in this gene likely will be associated with more mild respiratory disease that may fail to be diagnosed as PCD, as individuals with such mutations would not present with the severe disease and may be difficult to diagnose. This may be why it has not yet been reported in the clinical literature.

One limitation of our study is the ex vivo nature of the current $\mu \mathrm{OCT}$ system, which, for human patients, is limited to visualizing primary airway cells in culture or potentially excised tissues. Miniaturization of the $\mu \mathrm{OCT}$ probe may allow in vivo imaging of ciliary motion in the human nasal passages for rapid noninvasive assessment of ciliary dynamics for PCD diagnosis in the future; initial attempts are already in progress (32). Another limitation is our use of murine models, which do not express the glandular structures of the human airway, which alters mucus content. In our analysis, we standardized fluid load by measuring MCT following application of a uniform fluid load to minimize secondary variations due to mucus viscosity. Nevertheless, higher-order mammals may be able to more appropriately model these features. It should be noted that, while $\mu \mathrm{OCT}$ provides in situ measurements that are key to understanding the severity and context of the defect, HSVM remains highly useful, especially for visualizing the individual cilium due to its superior resolution at present. Although the current $\mu \mathrm{OCT}$ system described here provides a number of metrics surrounding ciliary motion and stroke that provide a comprehensive view of the PCD defect, more simple imaging systems, such as conventional OCT (33) or even HSVM, can also provide metrics surrounding CBF (14) and waveforms (21), which could be informative even as a single measure and may 

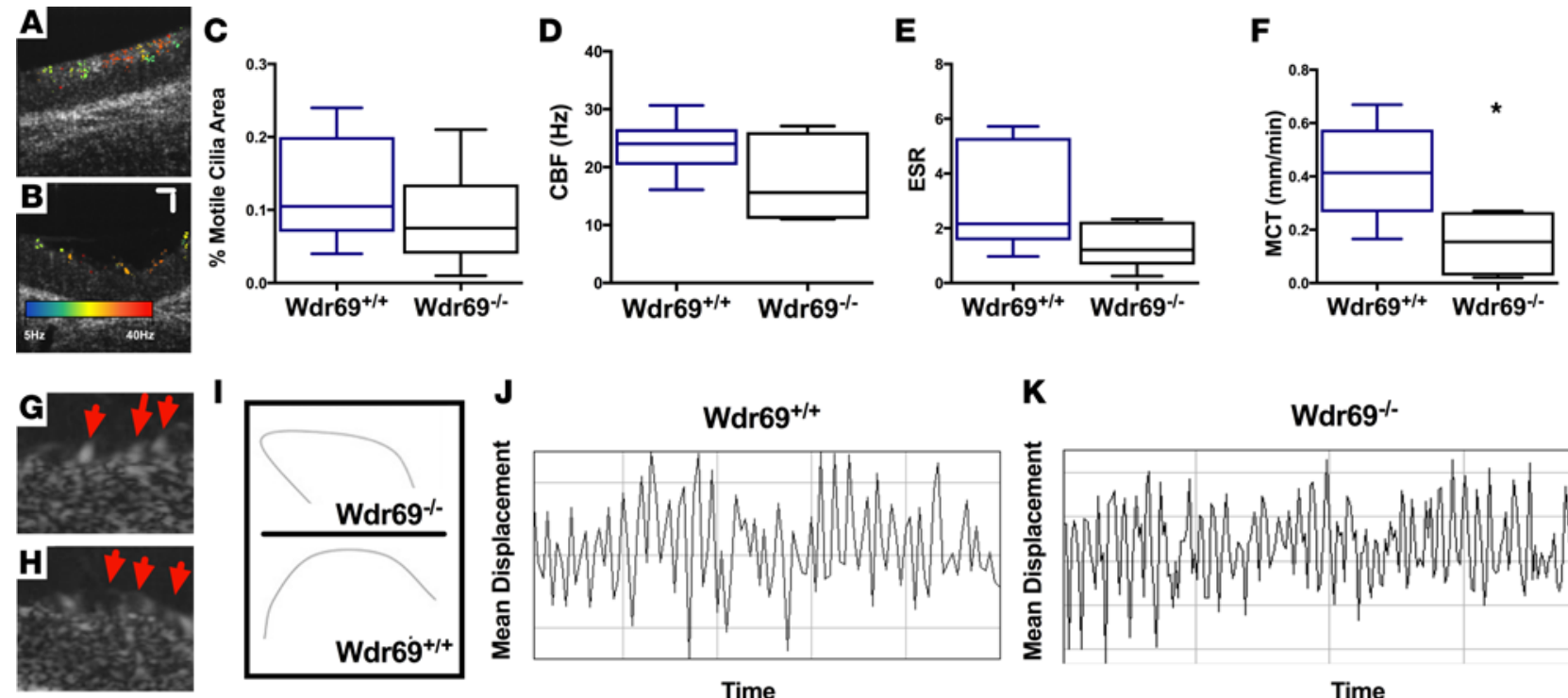
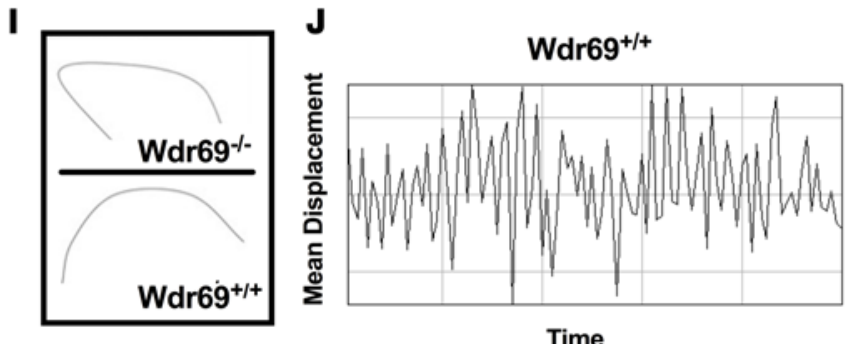

$\mathbf{L}$

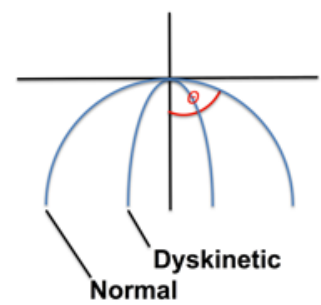

M

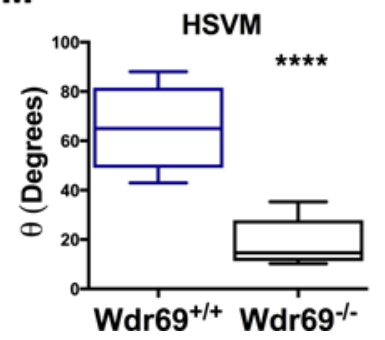

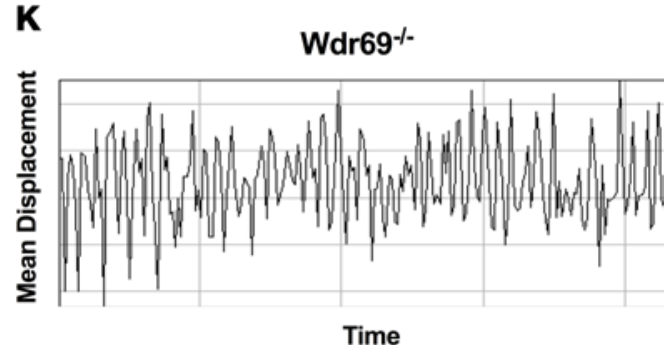

$\mathbf{N}$

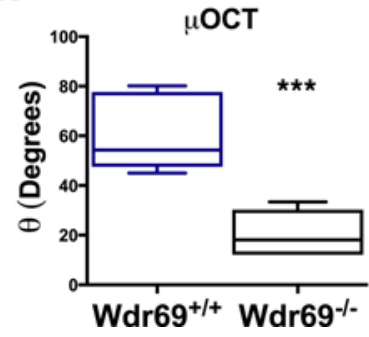

Figure 5. Characterization of $\mathbf{W d r 6 9 ^ { - / - }}$ mice. (A) Representative motile ciliary area map from Wdr69+/+ littermate control and (B) Wdr69 ${ }^{-/-}$mice indicating equivalent motile cilia areas. Colored pixels indicate areas of active cilia movement at varied CBFs. (C) Quantification of equivalent percentages of motile cilia areas in Wdr695//- mice and Wdr69 ${ }^{+/+}$littermate control mice. (D) CBF rates are equivalent in Wdr695//- mice and Wdr69+/+ littermate control mice. (E) Equivalent coverage and CBF indicates that reduced ESR in Dnah5 ${ }^{-1-}$ mice is not responsible for reduced MCT. (F) There was marked reduction in MCT in the Wdr69-/- mice compared with controls. ${ }^{*} P<0.05$. (G-I) Examination of cilia arcs made possible by $\mu$ OCT analysis demonstrated characteristic dyskinesia and narrow "flame" pattern of cilia arcs (red arrows) in Wdr695/- mice (G) compared with Wdr69+/+ mice (H), with graphical displays of this dyskinesia (I). (J and K) Representative waveform plots from Wdr69 $9^{+/+}$and Wdr69-/- mice, respectively, indicating equivalent ciliary activity at the ROI examined. (L) This analysis helped develop the arc angle measurement, defined as the angle between the edge of the cilia arc and artificial coordinates drawn bisecting the cilia arc through its apex. (M and $\mathbf{N}$ ) Cilia arc angles were reduced in Wdr69 $9^{-/-}$mice using both HSVM (M) and $\mu$ OCT ( $\mathbf{N}$ ), explaining reduced MCT in this mutation of outer dynein arm assembly and expression. ${ }^{* *} P<0.005 . n=8 \mathrm{Wdr69^{+/+ }}$ animals and $8 \mathrm{WDR69-/-}$ animals; 4 ROIs per trachea. All data are represented as mean (line) with minimum and maximum boundaries displayed. Statistical significance determined by Mann-Whitney $t$ test. Scale bar: 10 microns (two dimensions, $\mathbf{A}$ and $\mathbf{B}$ ); $\mathbf{G}$ and $\mathbf{H}$ are zoomed $2 \mathrm{X}$ of $\mathbf{A}$ and $\mathbf{B}$.

be simple to implement in vivo. We acknowledge that the current $\mu \mathrm{OCT}$ imaging system allows only for visualization of planar motion. $\mathrm{CBF}$ measured here are somewhat higher than those reported in the literature, likely because $\mathrm{CBF}$ is higher in very young mice (34) and is extremely sensitive to temperature; our studies were all conducted at $37^{\circ} \mathrm{C}$ and, in some cases, in pups within the first week of life. Although the ciliary effective stroke of motile respiratory cilia is thought to be mostly planar in two dimensions (35-37), three-dimensional analysis to refine the view of ciliary motion could reveal additional deficits not readily observed at present using $\mu$ OCT. Future iterations of $\mu$ OCT that will allow for three-dimensional imaging of individual cilia are in development. We also acknowledge that the MCT measured was standardized and represents a fluid flow rate due to the observation of a standardized fluid added to the epithelial surface. This experimental design was necessary to ensure that ciliary motion, and not secondary changes in mucus adhesion and/or mucus viscosity, accounted for differences in fluid propulsion.

Our study provides evidence for an imaging modality that offers an integrated analysis of ciliary motion and MCT using in situ measurements, providing a key measure of contextual severity and a number of potential applications in the future. Our findings show the potential utility of $\mu$ OCT to provide quantitative assessments that can elucidate the functional basis for the ciliary motion abnormality and its effect 
A

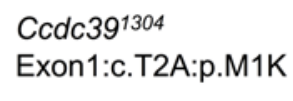

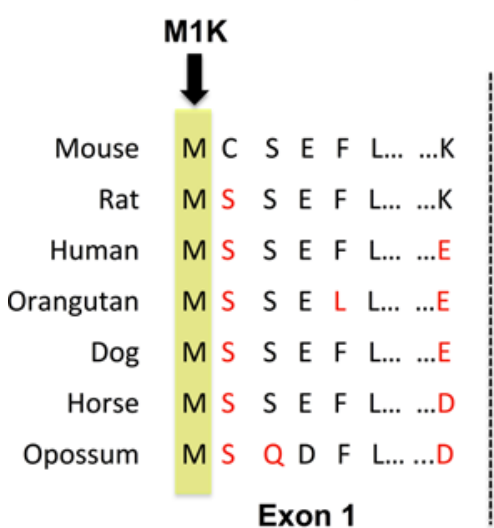

B Wdr69 Nebulous

Exon3:c.T197C:p.L66P

L66P

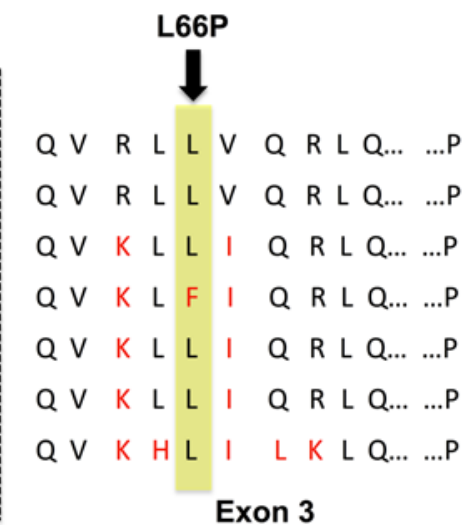

C Dnah5 Dakshi

Exon59:c.T10048C:p.S335P

S3350P

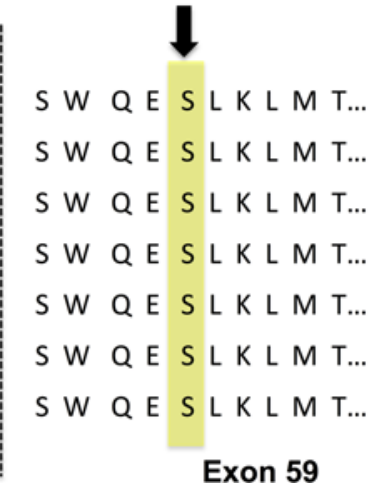

Figure 6. Homology of PCD genes examined in higher-order mammalian species.

on MCT. This may be useful not only for PCD, but also for evaluation of a variety of respiratory conditions that are presently without adequate explanation. The recent demonstration of the genetic heterogeneity of PCD and the wide spectrum of ciliary motion defects seen in these patients have highlighted the need for new approaches to assess ciliary motion functionally to determine the risk for respiratory disease and other organ dysfunction. Ultimately, $\mu \mathrm{OCT}$ may have the capacity to help predict the treatments most likely to be efficacious based on the underlying basis of the ciliary motion abnormality, while also allowing monitoring of the effect of novel therapeutic treatments on the mucociliary apparatus.

\section{Methods}

Mutant mouse models. Male and female mice harboring mutations in Dnah5 (at age 15-25 days), Ccdc39 (at age 5-7 days), and $W d r 69$ (at age 30-70 days) were recovered from a large-scale mouse mutagenesis screen using ethylnitrosourea (38). The mutations generated in the model demonstrate homology to a diverse range of higher mammalian species. The mutations were generated and maintained in a C57BL/6J (B6) background, with more than 8 generations of B6 backcrossing to remove incidental mutations. All the mutants are cryopreserved at the Jackson Laboratory and have been annotated and curated as part of the Bench to Bassinet collection of mutant mice in the Mouse Informatics Database (see Figure 6). These data demonstrate homology of the mutations recovered in the murine lines to higher-order mammals, including humans, adding relevance to the models studied to predict abnormalities in human PCD.

Preparation of tracheal tissue. Mutant mice were euthanized when moribund from hydrocephalus; Dnah5 mice were euthanized at age 15-25 days, $C c d c 39$ mice at age $<7$ days, and $W d r 69$ mice at age 30-70 days, along with age-matched, littermate, wild-type controls. The tracheae were removed for OCT analysis of respiratory ciliary motion and mucus clearance. After euthanasia, trachea were removed, separated from the distal airways and lung tissue, placed in HEPES-buffered DMEM (Life Technologies), and shipped to the University of Alabama at Birmingham at $4^{\circ} \mathrm{C}$ overnight. Upon receipt, tracheal tissue was dissected longitudinally along the posterior membranous tracheal section to expose the luminal epithelial cell surface and incubated for 30 minutes under physiologic conditions $\left(37^{\circ} \mathrm{C}, 5 \% \mathrm{CO}_{2}, 100 \%\right.$ humidity) at air-liquid interface to remove the residual transport media and allow the epithelial surface to equilibrate.

$\mu O C T$ imaging. Functional assessments of the tracheal tissue explants were performed at air-liquid interface using $\mu \mathrm{OCT}$ as previously described (25) in an environmental chamber with a temperature regulatory device (Incubator PM 2000 RBT with TempModule S, Carl Zeiss), with the acquisition speed set at 20,480 Hz line rate to yield 80 or 100 frames per second at 256 or 204 lines per frame, respectively. Playback rate was 40 fps. Images were recorded at $5-10$ ROI per piece of tissue (each ROI is $240-\mu \mathrm{m}$ wide) per condition on the anterior surface of the midtrachea where there is adequate and consistent ciliated cell expression. Images were recorded at randomly chosen intervals on the anterior mucosal surface of the midtrachea, with the optical beam scanned along the longitudinal direction as previously described $(25,39)$ by a blinded imager. After images were taken with native mucus, $1 \mu \mathrm{l}$ of $500 \mathrm{~nm}$ standardized fluorescent bead solution $\left(1 \times 10^{8}\right.$ particles $/ \mathrm{ml}$ diluted 1:100 
v/v with PBS) was added to the anterior surface of the tracheal lumen to simulate a standard fluid workload with particles to minimize the confounding effects of variable adherence and viscosity of native mucus. Thus, MCT measured throughout is the measurement of standardized fluid flow rate described here.

HSVM imaging. For HSVM imaging, tracheal samples were removed and dissected as described above and as previously reported (34). Tracheal samples were then transferred to a glass-bottom culture dish with L-15 media and covered with a housing composed of hollowed silicone gel and topped with a glass coverslip as previously described (34). Cilia HSVM imaging was carried out using DIC imaging on a Leica DMRE inverted microscope with a $\times 100$ oil objective (NA: 1.40 ) and a Phantom v4.2 camera (Leica) operated at 200 frames per second. Playback rate was set at $30 \mathrm{fps}$.

$\mu O C T$ image analysis. All tissues were imaged with the beam directly perpendicular to the long axis of the trachea, with tracheal tissue measured in the caudal to cranial direction so that the active cilia plane (40-43) was coincident with the OCT beam; the beam was oriented perpendicular to the long axis of the trachea with MCT in the caudal to cranial direction (40-43). Several metrics were quantified from $\mu \mathrm{OCT}$-recorded images using ImageJ (NIH) and Matlab. Airway surface liquid (ASL) and periciliary layer depths were measured directly (see Figure 1A) as previously described (25) using ImageJ. MCT rate was calculated based on the average slope of the distance per time of standardized bead particulates in the mucus over several frames in the ASL region up to $50 \mu \mathrm{m}$ above the epithelial cell surface (see Figure 1E) as previously described $(25,33,44)$. CBF was determined by temporally high-pass filtering the images at $6 \mathrm{~Hz}$ to remove low-frequency vibrations, computing the Fourier power spectrum for all $3 \times 3$ pixel subregions in the image, and selecting the subregions ranking highest in peak sharpness, defined as the peak power density divided by the total power density (25). Measurements from up to 10 subregions were aggregated for each ROI automatically, with values corresponding to spurious peaks manually rejected in cases of abnormal power spectral density appearance, such as multiple peak frequencies in one subregion.

Coverage of active cilia (percentage motile cilia area) was determined using a map of CBF density across the epithelial cell surface (see Figure 1B) (44). Each video was assessed for the presence of cilia in motion. Areas of ciliary activity were determined from $\mu \mathrm{OCT}$ images by identifying regions of the image with periodic oscillations in intensity that exceed random variations due to noise, defined for this purpose as 4 times the median standard deviation of each pixel in the image. The motile cilia area was then defined by the ratio of the number of lines with cilia motion to the number without motile cilia in each video. Cilia coverage maps were generated by overlaying a color on pixels identified as active cilia, where the color was a function of the peak frequency identified on the power spectrum. The frequencies identified in cilia maps were utilized for visualization purposes only; computation of $\mathrm{CBF}$ for a given region was subject to spatial and temporal filtering to reduce the effect of noise, as described in the preceding paragraph.

Other analytics included measurement of the arc angle, defined as the angular displacement of the cilia during its effective stroke (see Figure 1C). This metric was measured as degree of the angle formed by arbitrary coordinates of cilia arc apex and the lateral edge of the cilia arc at its full extent in time averaged across images of each video (4-6 videos per tracheal sample), based on the principle that the effective and recovery strokes are essentially planar in two dimensions $(8,16)$. Angular measurements of cilia arcs in each tracheal image were analyzed using angular measurement tools in Image $J$ of $\mu \mathrm{OCT}$ and were confirmed with HSVM (Supplemental Figure 1). Wild-type and disease mutant ARCs were measured by a blinded reviewer of HSVM and $\mu \mathrm{OCT}$ using ImageJ, with an excellent correlation between ARCs measured by the two different imaging modalities ( $\mathrm{r}=0.9868, P<0.0005$, Supplemental Figure 1$)$. The linear regression equation for this relationship was found as follows: $\mathrm{ARC}_{\mu \mathrm{OCT}}=0.9495\left(\mathrm{ARC}_{\mathrm{HSVM}}\right)+1.553$.

Stroke kinetics $(\mathrm{CBF})$ and motile cilia area were integrated in a measurement of ESR, defined as the product of mean $\mathrm{CBF}$ and coverage (motile ciliated area) in a ROI. ESR accounts for integrated ciliary motion that is independent of other important factors on the epithelial surface, including coordinated ciliary motion (metachrony) and mucus viscosity.

Tissues from younger animals demonstrate some reduction in resolution due to the thinner nature of murine tracheal tissue prior to adulthood, which results in reduced interference between epithelial layers. Statistics. All measurements were analyzed by a reviewer blinded to the genotype of animals. All data are presented as the mean $( \pm S E M)$. Each tracheal sample data point represents the means of 5-10 ROI from the sample. All quantitative data were analyzed using descriptive statistics calculated in Graph Pad Prism 6.0 (GraphPad Software Inc.). Statistical significance was defined as $P<0.05$ using nonparametric 2-tailed Mann-Whitney $t$ test (due to small data sets). 
Study approval. All animal studies were approved the University of Pittsburgh Institutional Animal Care and Use Committee under protocol IS00005836.

\section{Author contributions}

GMS, SMR, GJT, and CWL contributed to study design. GMS, RF, KKC, SEB, GG, JET, KLL, NK, and BT performed experiments. GMS, KKC, SEB, GJT, CWL, and SMR analyzed and interpreted the data. GMS, GJT, CWL, and SMR contributed to manuscript preparation. All authors approved of the final version of the manuscript.

\section{Acknowledgments}

This work is supported by grants from the NIH (P30 DK072482 to SMR, HL1116213 to SMR and GJT, and HL098180 and GM104412 to CWL), the Cystic Fibrosis Foundation (CLANCY09Y0 to GMS and SORSCH15RO to GMS), and the Mucociliary Clearance Consortium (to SMR and GJT) funded by Cystic Fibrosis Foundation Therapeutics.

Address correspondence to: Steven M. Rowe, MCLM 7061918 University Boulevard, Birmingham, Alabama 35294-0006, USA. Phone: 205.934.9640; E-mail: smrowe@uab.edu.

1. Bush A, et al. Primary ciliary dyskinesia: current state of the art. Arch Dis Child. 2007;92(12):1136-1140.

2. Knowles MR, Daniels LA, Davis SD, Zariwala MA, Leigh MW. Primary ciliary dyskinesia. Recent advances in diagnostics, genetics, and characterization of clinical disease. Am J Respir Crit Care Med. 2013;188(8):913-922.

3. Leigh MW, et al. Clinical and genetic aspects of primary ciliary dyskinesia/Kartagener syndrome. Genet Med. 2009;11(7):473-487.

4. Morillas HN, Zariwala M, Knowles MR. Genetic causes of bronchiectasis: primary ciliary dyskinesia. Respiration. 2007;74(3):252-263.

5. Kennedy MP, et al. High-resolution CT of patients with primary ciliary dyskinesia. AJR Am J Roentgenol. 2007;188(5):1232-1238

6. Sagel SD, Davis SD, Campisi P, Dell SD. Update of respiratory tract disease in children with primary ciliary dyskinesia. Proc Am Thorac Soc. 2011;8(5):438-443.

7. Noone PG, et al. Primary ciliary dyskinesia: diagnostic and phenotypic features. Am J Respir Crit Care Med. 2004;169(4):459-467.

8. O'Callaghan C, Sikand K, Rutman A. Respiratory and brain ependymal ciliary function. Pediatr Res. 1999;46(6):704-707.

9. Greenstone MA, Jones RW, Dewar A, Neville BG, Cole PJ. Hydrocephalus and primary ciliary dyskinesia. Arch Dis Child. 1984;59(5):481-482.

10. McComb P, Langley L, Villalon M, Verdugo P. The oviductal cilia and Kartagener's syndrome. Fertil Steril. 1986;46(3):412-416.

11. Sha YW, Ding L, Li P. Management of primary ciliary dyskinesia/Kartagener's syndrome in infertile male patients and current progress in defining the underlying genetic mechanism. Asian J Androl. 2014;16(1):101-106.

12. Li Y, et al. Global genetic analysis in mice unveils central role for cilia in congenital heart disease. Nature. 2015;521(7553):520-524

13. Leigh MW, O'Callaghan C, Knowles MR. The challenges of diagnosing primary ciliary dyskinesia. Proc Am Thorac Soc. 2011;8(5):434-437.

14. Quinn SP, Zahid MJ, Durkin JR, Francis RJ, Lo CW, Chennubhotla SC. Automated identification of abnormal respiratory ciliary motion in nasal biopsies. Sci Transl Med. 2015;7(299):299ra124.

15. Chilvers MA, Rutman A, O'Callaghan C. Functional analysis of cilia and ciliated epithelial ultrastructure in healthy children and young adults. Thorax. 2003;58(4):333-338.

16. O'Callaghan C, Sikand K, Chilvers MA. Analysis of ependymal ciliary beat pattern and beat frequency using high speed imaging: comparison with the photomultiplier and photodiode methods. Cilia. 2012;1(1):8.

17. Papon JF, et al. Quantitative analysis of ciliary beating in primary ciliary dyskinesia: a pilot study. Orphanet J Rare Dis. 2012;7:78.

18. O'Callaghan C, Rutman A, Williams GM, Hirst RA. Inner dynein arm defects causing primary ciliary dyskinesia: repeat testing required. Eur Respir J. 2011;38(3):603-607.

19. Knowles MR, Leigh MW, Zariwala MA. Cutting edge genetic studies in primary ciliary dyskinesia. Thorax. 2012;67(5):464; author reply 464.

20. Knowles MR, et al. Mutations of DNAH11 in patients with primary ciliary dyskinesia with normal ciliary ultrastructure. Thorax. 2012;67(5):433-441.

21. Raidt J, et al. Ciliary beat pattern and frequency in genetic variants of primary ciliary dyskinesia. Eur Respir J. 2014;44(6):15791588.

22. Li Y, et al. Respiratory motile cilia dysfunction in a patient with cranioectodermal dysplasia. Am J Med Genet A. 2015;167A(9):2188-2196.

23. Garrod AS, et al. Airway ciliary dysfunction and sinopulmonary symptoms in patients with congenital heart disease. Ann Am Thorac Soc. 2014;11(9):1426-1432.

24. Salathe M. Regulation of mammalian ciliary beating. Annu Rev Physiol. 2007;69:401-422.

25. Liu L, et al. Method for quantitative study of airway functional microanatomy using micro-optical coherence tomography. PLoS One. 2013;8(1):e54473.

26. Antony D, et al. Mutations in CCDC39 and CCDC40 are the major cause of primary ciliary dyskinesia with axonemal disorganization and absent inner dynein arms. Hum Mutat. 2013;34(3):462-472. 
27. Merveille AC, et al. CCDC39 is required for assembly of inner dynein arms and the dynein regulatory complex and for normal ciliary motility in humans and dogs. Nat Genet. 2011;43(1):72-78.

28. Davis SD, et al. Clinical features of childhood primary ciliary dyskinesia by genotype and ultrastructural phenotype. Am $J$ Respir Crit Care Med. 2015;191(3):316-324.

29. Olbrich $\mathrm{H}$, et al. Mutations in DNAH5 cause primary ciliary dyskinesia and randomization of left-right asymmetry. Nat Genet. 2002;30(2):143-144.

30. Gao C, Wang G, Amack JD, Mitchell DR. Oda16/Wdr69 is essential for axonemal dynein assembly and ciliary motility during zebrafish embryogenesis. Dev Dyn. 2010;239(8):2190-2197.

31. Ahmed NT, Gao C, Lucker BF, Cole DG, Mitchell DR. ODA16 aids axonemal outer row dynein assembly through an interaction with the intraflagellar transport machinery. J Cell Biol. 2008;183(2):313-322.

32. Chu KK, et al. In vivo imaging of airway cilia and mucus clearance with micro-optical coherence tomography. Biomed Opt Express. 2016;7(7):2494-2505.

33. Oldenburg AL, Chhetri RK, Hill DB, Button B. Monitoring airway mucus flow and ciliary activity with optical coherence tomography. Biomed Opt Express. 2012;3(9):1978-1992.

34. Francis R, Lo C. Ex vivo method for high resolution imaging of cilia motility in rodent airway epithelia. J Vis Exp. 2013;(78): 50343

35. Sanderson MJ, Sleigh MA. Ciliary activity of cultured rabbit tracheal epithelium: beat pattern and metachrony. J Cell Sci. 1981;47:331-347.

36. Marshall WF. Cilia self-organize in response to planar cell polarity and flow. Nat Cell Biol. 2010;12(4):314-315.

37. Ginger ML, Portman N, McKean PG. Swimming with protists: perception, motility and flagellum assembly. Nat Rev Microbiol. 2008;6(11):838-850.

38. Tan SY, et al. Heterotaxy and complex structural heart defects in a mutant mouse model of primary ciliary dyskinesia. JClin Invest. 2007;117(12):3742-3752.

39. Birket SE, et al. A functional anatomic defect of the cystic fibrosis airway. Am J Respir Crit Care Med. 2014;190(4):421-432.

40. Chien YH, et al. Bbof1 is required to maintain cilia orientation. Development. 2013;140(16):3468-3477.

41. Rautiainen M, Collan Y, Nuutinen J. A method for measuring the orientation ("beat direction") of respiratory cilia. Arch Otorhinolaryngol. 1986;243(4):265-268.

42. Delaere PR, Liu Z, Delanghe G, Gyselen K, Jorissen M, Feenstra L. Mucociliary clearance following segmental tracheal reversal. Laryngoscope. 1996;106(4):450-456

43. Sleigh MA. Movement and coordination of tracheal cilia and the relation of these to mucus transport. Prog Clin Biol Res. 1982;80:19-24.

44. Zhou KC, Huang BK, Gamm UA, Bhandari V, Khokha MK, Choma MA. Particle streak velocimetry-optical coherence tomog raphy: a novel method for multidimensional imaging of microscale fluid flows. Biomed Opt Express. 2016;7(4):1590-1603. 